\title{
Testing microsatellite instability in solid tumors: the ideal versus what is real
}

\author{
Virgilio Souza E. Silva ${ }^{1}$, Louise De Brot ${ }^{2}$, Rachel P. Riechelmann ${ }^{1}$ \\ ${ }^{1}$ Department of Clinical Oncology, ${ }^{2}$ Department of Pathology, AC Camargo Cancer Center, Sao Paulo, Brazil \\ Correspondence to: Rachel P. Riechelmann. Department of Clinical Oncology, AC Camargo Cancer Center, Rua Antonio Prudente 211, CEP 01509- \\ 010, São Paulo, SP, Brazil. Email: rachel.riechelmann@accamargo.org.br. \\ Provenance: This is an invited article commissioned by the Academic Editor Dr. Jingping Wang (Department of Pathology, The First Affiliated \\ Hospital of Bengbu Medical College, Bengbu Medical College, Bengbu, China). \\ Comment on: Cohen R, Hain E, Buhard O, et al. Association of Primary Resistance to Immune Checkpoint Inhibitors in Metastatic Colorectal Cancer \\ With Misdiagnosis of Microsatellite Instability or Mismatch Repair Deficiency Status. JAMA Oncol 2019;5:551-5.
}

Submitted Sep 10, 2019. Accepted for publication Sep 20, 2019.

doi: $10.21037 /$ atm.2019.09.124

View this article at: http://dx.doi.org/10.21037/atm.2019.09.124

The mismatch repair (MMR) genes $M L H 1$, MSH2, MSH6, and $P M S 2$ are responsible for correcting the nucleotide base mispairings and small insertions or deletions that occur during DNA replication. Defects of the MMR system, resulting from mutations in any of the MMR genes, lead to microsatellite instability (MSI) (1), which is observed in approximately $2 \%$ to $3 \%$ of solid tumors, particularly in metastatic colorectal cancer (mCRC) and other Lynch Syndrome related tumors, such as endometrial carcinoma $(2,3)$. The deficient MMR system results in tumors with a large number of mutations, and consequently the surge of neoantigens and the so-called "immune phenotype". MMR deficiency is one of the strongest predictive biomarkers of response to treatment with immune checkpoint inhibitors (ICI) $(4,5)$.

The hypothesis that MSI in mCRC are responsive to ICI treatment has been addressed in recent studies that demonstrated high response rates and durable clinical benefit (6). This provides the rationale for routine testing of MSI and/or deficient (d) MMR in mCRC. Thus, the accuracy of the MSI and/or dMMR assessment is critical to avoid misdiagnosis and inappropriate indications of treatment with immunotherapy.

In FAMA Oncology, Cohen et al. (7) described the results of a retrospective analysis of 38 patients with MSI and/ or dMMR mCRC treated with ICI. They showed that primary resistance to ICI was observed in 5 individuals $(13 \%)$, where $3(60 \%)$ were misdiagnosed as having MSI or dMMR tumors but actually had microsatellite stable (MSS)
mCRC. In the same study, a retrospective multicentric cohort of 93 patients with mCRC validated the previous findings: a misdiagnosis rate by local assessment of $10 \%$ (9 of the 93 mCRC tumors showing both proficient MMR and MSS). The authors concluded that primary resistance to ICI in patients with mCRC displaying MSI or dMMR mostly reflects incorrect assessment of the MSI/dMMR status. Based on their findings, the authors recommended routine testing with both immunohistochemistry (IHC) and polymerase chain reaction (PCR) to confirm the MSI status prior to ICI treatment. The European Society of Medical Oncology (ESMO) guideline also recommends the use of both MMR-IHC and MSI-PCR to assess the eligibility to ICI (8). While the evidence points to the use of both tests, some issues have to be addressed before the scientific community decides to endorse this strategy.

As a molecular indicator of the defective DNA MMR system, MSI can be determined by loss of expression of any of the MMR proteins by IHC or by molecular assays such as MSI-PCR (9). IHC is easier to perform, has broader availability, is less expensive (10), performed rapidly, points to the defective $M M R$ gene and is considerably reliable (11), gaining strength as a standard method to diagnose $\mathrm{d} M M R$. Lindor et al. (12) reported that IHC with MLH1/MSH2 antibodies had $100 \%$ specificity and $92 \%$ sensitivity for MMR proficiency in colorectal cancer. Results of other studies showed that the inclusion of PMS2 and MSH6 to the panel of antibodies further increased the sensitivity of IHC to predict MSI and germ-line mutations in colorectal 
cancer equivalent to that of MSI testing $(13,14)$.

Yet, there are some limitations of IHC to determine MMR status. These include variations in staining patterns that could impair the pathologist interpretation of $\mathrm{dMMR}$. Therefore, improvement in the IHC testing process could lead to its greater accuracy as a first screening test for $\mathrm{dMMR}$. Better tissue acquirement and processing are crucial steps in this process, and results of MMR testing are directly linked to the quality of tissue. While tissue obtained after cytotoxic and/or radiation therapy tends to be of lower quality, treatment-naïve tumor is ideal for IHC. Also, tumor tissues have to be obtained in large chunks and preserved in buffered formaldehyde for a definite amount of time so it can be properly fixed while not losing its immunogenic properties for the IHC reaction (15). Additionally, the role of the pathologist here is pivotal. First the pre-analytic part needs to be supervised, to assure sufficient quality prior to the IHC reactions; training is fundamental to adequately interpret IHC results as truly positive or negative, since some patters of staining can be misleading (16). In this setting, a false-negative result would prevent a patient to derive benefit from ICI and a false-positive, could lead adverse events without efficacy but with significant financial toxicity (17).

Despite IHC sufficiency as a biomarker for ICI usage, molecular testing remains a gold standard for a final definition of MMR status. The MSI-PCR molecular test is a amplification-based test evaluated by a panel of five microsatellite markers with poly-A mononucleotide repeats (MTD-25, MTD-26, NR-21, NR-24, NR-27) (18,19). The advantages of the PCR-MSI test are its ability to detect MSI caused by $M M R$ genes not tested by IHC, such as EPCAM (20), and to identify the rare cases of MSI secondary to non-truncating missense mutations that present with intact IHC staining for MMR proteins (21). Although more expensive, MSI molecular testing requires one tumor section, while IHC needs at least four (22). On the other hand, MSI-PCR cannot detect cases of MSS hypermutated tumors, such as in POLE mutations or in tumors with high tumor mutational burden by unknown mechanisms (23). The addition of IHC and MSI-PCR surely adds in accuracy, but even when combined, they have some intrinsic limitations on predicting response to ICI.

While we agree with Cohen et al. and the ESMO guideline that using both IHC and MSI-PCR is adequate for the great majority of cases, we also realize that it is not feasible globally to depend on both methods, especially due to lack of access to MSI-PCR. On the other hand, when resources are available, genetic panels are the preferred method to evaluate not only the molecular profiles of mCRC but also to look for MSI/MMR gene mutations and mutation load.

In real-life situations diagnosis of MSI/dMMR mCRC has to be carefully deliberated with the available tools in order to maximize benefit to patients. It is expected that the usage of isolated IHC to screen for dMMR would be enough for indicating ICI treatment, with the MSI-PCR molecular test being indicated in cases of undetermined IHC results. Efforts should definitely be made to maximize the quality of available testing, improving the quality of tumor tissue acquirement and preservation, minimizing preanalytical issues, and, finally proper selection of treatmentnaïve tumor materials to maximize the accuracy of the IHC test. With all efforts directed to upgrade the MSI/dMMR testing accuracy in all possible means, the benefit from ICI treatment will undoubtedly be pumped up.

\section{Acknowledgments}

None.

\section{Footnote}

Conflicts of Interest: The authors have no conflicts of interest to declare.

Ethical Statement: The authors are accountable for all aspects of the work in ensuring that questions related to the accuracy or integrity of any part of the work are appropriately investigated and resolved.

\section{References}

1. Lynch HT, Snyder CL, Shaw TG, et al. Milestones of Lynch syndrome: 1895-2015. Nat Rev Cancer 2015;15:181-94.

2. Venderbosch S, Nagtegaal ID, Maughan TS, et al. Mismatch Repair Status and BRAF Mutation Status in Metastatic Colorectal Cancer Patients: A Pooled Analysis of the CAIRO, CAIRO2, COIN, and FOCUS Studies. Clin Cancer Res 2014;20:5322-30.

3. Modica I, Soslow RA, Black D, et al. Utility of immunohistochemistry in predicting microsatellite instability in endometrial carcinoma. Am J Surg Pathol 2007;31:744-51.

4. Le DT, Uram JN, Wang H, et al. PD-1 Blockade in 
Tumors with Mismatch-Repair Deficiency. N Engl J Med 2015;372:2509-20.

5. Dudley JC, Lin MT, Le DT, et al. Microsatellite Instability as a Biomarker for PD-1 Blockade. Clin Cancer Res 2016;22:813-20.

6. Gelsomino F, Barbolini M, Spallanzani A, et al. The evolving role of microsatellite instability in colorectal cancer: A review. Cancer Treat Rev 2016;51:19-26.

7. Cohen R, Hain E, Buhard O, et al. Association of Primary Resistance to Immune Checkpoint Inhibitors in Metastatic Colorectal Cancer With Misdiagnosis of Microsatellite Instability or Mismatch Repair Deficiency Status. JAMA Oncol 2019;5:551-5.

8. Luchini C, Bibeau F, Ligtenberg MJ, et al. ESMO recommendations on microsatellite instability testing for immunotherapy in cancer, and its relationship with PD-1/ PD-L1 expression and tumour mutational burden: a systematic review-based approach. Ann Oncol 2019. [Epub ahead of print].

9. Umar A, Boland CR, Terdiman JP, et al. Revised Bethesda Guidelines for Hereditary Nonpolyposis Colorectal Cancer (Lynch Syndrome) and Microsatellite Instability. J Natl Cancer Inst 2004;96:261-8.

10. Debniak T, Kurzawski G, Gorski B, et al. Value of pedigree/ clinical data, immunohistochemistry and microsatellite instability analyses in reducing the cost of determining $\mathrm{hMLH} 1$ and $\mathrm{hMSH} 2$ gene mutations in patients with colorectal cancer. Eur J Cancer 2000;36:49-54.

11. Shia J. Immunohistochemistry versus Microsatellite Instability Testing For Screening Colorectal Cancer Patients at Risk For Hereditary Nonpolyposis Colorectal Cancer Syndrome: Part I. The Utility of Immunohistochemistry. J Mol Diagn 2008;10:293-300.

12. Lindor NM, Burgart LJ, Leontovich O, et al. Immunohistochemistry Versus Microsatellite Instability Testing in Phenotyping Colorectal Tumors. J Clin Oncol 2002;20:1043-8.

13. de Jong AE, van Puijenbroek $M$, Hendriks $Y$, et al. Microsatellite instability, immunohistochemistry, and additional PMS2 staining in suspected hereditary nonpolyposis colorectal cancer. Clin Cancer Res

Cite this article as: Silva VSE, De Brot L, Riechelmann RP. Testing microsatellite instability in solid tumors: the ideal versus what is real. Ann Transl Med 2019;7(21):600. doi: 10.21037/ atm.2019.09.124
2004;10:972-80.

14. Halvarsson B, Lindblom A, Rambech E, et al. The added value of PMS2 immunostaining in the diagnosis of hereditary nonpolyposis colorectal cancer. Fam Cancer 2006;5:353-8.

15. Piñol V, Castells A, Andreu M, et al. Accuracy of Revised Bethesda Guidelines, Microsatellite Instability, and Immunohistochemistry for the Identification of Patients With Hereditary Nonpolyposis Colorectal Cancer. JAMA 2005;293:1986-94.

16. Kim SW, Roh J, Park CS. Immunohistochemistry for Pathologists: Protocols, Pitfalls, and Tips. J Pathol Transl Med 2016;50:411-8.

17. Tran G, Zafar SY. Financial toxicity and implications for cancer care in the era of molecular and immune therapies. Ann Transl Med 2018;6:166.

18. Goel A, Nagasaka T, Hamelin R, et al. An optimized pentaplex PCR for detecting DNA mismatch repairdeficient colorectal cancers. PLoS One 2010;5:e9393.

19. Buhard O, Cattaneo F, Wong YF, et al. Multipopulation Analysis of Polymorphisms in Five Mononucleotide Repeats Used to Determine the Microsatellite Instability Status of Human Tumors. J Clin Oncol 2006;24:241-51.

20. Kempers MJ, Kuiper RP, Ockeloen CW, et al. Risk of colorectal and endometrial cancers in EPCAM deletionpositive Lynch syndrome: a cohort study. Lancet Oncol 2011;12:49-55.

21. McCarthy AJ, Capo-Chichi JM, Spence T, et al. Heterogenous loss of mismatch repair (MMR) protein expression: a challenge for immunohistochemical interpretation and microsatellite instability (MSI) evaluation. J Pathol Clin Res 2019;5:115-29.

22. Zhang L. Immunohistochemistry versus Microsatellite Instability Testing for Screening Colorectal Cancer Patients at Risk for Hereditary Nonpolyposis Colorectal Cancer Syndrome: Part II. The Utility of Microsatellite Instability Testing. J Mol Diagn 2008;10:301-7.

23. Chalmers ZR, Connelly CF, Fabrizio D, et al. Analysis of 100,000 human cancer genomes reveals the landscape of tumor mutational burden. Genome Med 2017;9:34. 